\title{
Comparative Analysis of ANN-ICA and ANN-GWO for Crop Yield Prediction
}

\author{
Saeed Nosratabadi \\ School of the built Environment \\ Oxford Brookes University \\ Oxford, UK \\ 0000-0002-0440-6564 \\ Felde Imre \\ Kalman Kando Faculty of \\ Electrical Engineering \\ Obuda University \\ Budapest, Hungary \\ 0000-0003-4126-2480
}

\author{
Karoly Szell \\ Alba Regia Technical Faculty \\ Obuda University \\ Budapest, Hungary \\ 0000-0001-7499-5643
}

Sina Ardabili

Institute of advanced studies Koszeg

University of Pannonia

Koszeg, Hungary

0000-0002-7744-7906

\author{
Bertalan Beszedes \\ Alba Regia Technical Faculty \\ Obuda University \\ Budapest, Hungary \\ beszedes.bertalan@amk.uniobuda.hu \\ Amir Mosavi 1,2* \\ ${ }^{1}$ Department of Mathematics and \\ Informatics, J. Selye University \\ Komarno, Slovakia \\ ${ }^{2}$ Bauhaus Universität Weimar, \\ Weimar, Germany \\ 0000-0003-4842-0613
}

\begin{abstract}
Prediction of crops yield is essential for food security policymaking, planning, and trade. The objective of the current study is to propose novel crop yield prediction models based on hybrid machine learning methods. In this study the performance of artificial neural networks-imperialist competitive algorithm (ANN-ICA) and artificial neural networks-gray wolf optimizer (ANN-GWO) models for the crop yield prediction are evaluated. According to the results, ANNGWO, with R of 0.48, RMSE of 3.19, and MEA of 26.65, proved a better performance in the crop yield prediction compared to the ANN-ICA model. The results can be used by either practitioners, researchers or policymakers for food security.
\end{abstract}

Keywords-Hybrid machine learning, artificial neural networks, imperialist competitive algorithm, gray wolf optimization, crop yield

\section{INTRODUCTION}

Managing food security has turned into a very complicated and vital issue in the food supply chain [1]. Prediction of crop yield illustrates valuable possibilities for the management of food security in a food supply chain. Crop yield prediction presents the information that can be the basis of many important decisions related to food security, such as trading and developing policies [2].

On the other hand, forecasting the yield is not very easy as many controllable factors (e.g., applied irrigations, pest and fertilizer applications, etc.) and uncontrollable factors (e.g., weather, subsidies, and market, etc.) affect the crop yield [3, 4]. Numerous approaches were developed to predict the crop yield constituting farmers' long-term experience and the average of several previous yields. Whilst, Schlenker and Roberts [5] believe that the behavior of crop yield is not linear, and it varies from one year to another. The literature introduces data-driven models for crop yield production as the most accurate and efficient methods [6]. Although applying data-driven models for the crop yield prediction make accurate the data collection methods mechanisms, they are inexpensive and relatively easy to apply [6].

Therefore, the current study aims to find the most proper machine learning for crop yield prediction. To do so, a comparison study conducted to evaluate the performance of two hybrid machine learning methods artificial neural network imperialist competitive algorithm and artificial neural network gray wolf optimizer. This study took place in a large irrigated area in Kerman, Iran. In the following sections, the methodology and data collection process are described firstly. Then the performance of the mentioned machine learning methods is evaluated, and results and discussions consequently are provided.

\section{MATERIAL AND METHODS}

\section{A. Case Study}

The current study focused on the farms located near the city of "Kerman" in Iran. Kerman is the largest province in Iran (area of 183,285 km2), that embraces $11 \%$ of the land area of Iran (see Fig. 1). To meet the objective of the study, two types of data were collected: 1) agricultural production and 2) weather information. Spriter-GIS system used for the collection of data such as crop species, irrigation, and crop yield.

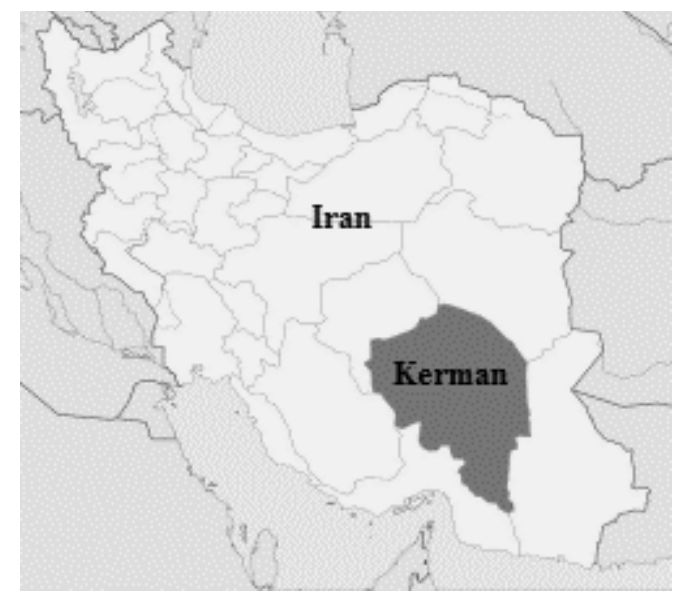

Fig. 1. Study area: Kerman, Iran

For the collection of data such as rainfall, solar radiation, and temperatures two meteorological stations placed on the 
site. On the other hand, agricultural products such as wheat, barley, potato, and sugar beets, which are the main productions of this region, were selected in this study. A summary of the collected data is presented in table I. As it is illustrated in table I, data recorded from 1998-2006 taken for further analysis in this study. The data related to 1998-2005 applied for the training phase and data 2005-2006 utilized for testing the methods' performance.

TABLE I. DISTRIBUTION OF SAMPLES DATASET IN TESTING AND TRAINING STAGES

\begin{tabular}{|c|c|c|c|c|}
\hline $\begin{array}{c}\text { Crop } \\
\text { species }\end{array}$ & $\begin{array}{c}\text { Total } \\
\text { number of } \\
\text { samples }\end{array}$ & $\begin{array}{c}\text { Samples in } \\
\text { the } \\
\text { training } \\
\text { period } \\
(\mathbf{1 9 9 9 -} \\
\mathbf{2 0 0 4}\end{array}$ & $\begin{array}{c}\text { Samples in } \\
\text { the testing } \\
\text { period } \\
(\mathbf{2 0 0 5} \\
\mathbf{2 0 0 6}\end{array}$ & $\begin{array}{c}\text { Testing } \\
\text { percentage } \\
\mathbf{( \% )}\end{array}$ \\
\hline Wheat & 508 & 449 & 59 & 11.61 \\
\hline Barley & 87 & 42 & 45 & 51.72 \\
\hline Potato & 195 & 132 & 63 & 32.31 \\
\hline $\begin{array}{c}\text { Sugar } \\
\text { Beet }\end{array}$ & 156 & 108 & 48 & 30.77 \\
\hline Total & 946 & 731 & 215 & \\
\hline
\end{tabular}

It is worth mentioning that the attributes considered in the current study were planting area (ha), irrigation water depth (mm) (which refers to total consumed water volume during the six stages of crop growth), rainfall during the crop growth stages $(\mathrm{mm})$, global solar radiation $\left(\mathrm{kWh} \mathrm{m}^{-2}\right)$ (point out to daily radiation in the last three crop growing stages), and maximum, average and minimum temperatures $\left({ }^{\circ} \mathrm{C}\right)$ which are recorded in the last three crop growing stages. It is aimed that finally to predict yield measured in $\left(\mathrm{t} \mathrm{ha}^{-1}\right)$.

\section{HYBRID MACHINE LEARNING METHODS}

As it is mentioned above, in this study, the performance of two hybrid machine learning methods in the crop yield prediction is compared. The selection of hybrid methods allows us to optimize the performance of machine learning in prediction [7]. According to Drummond et al. [8], ANN methods have had a very good performance in the crop yield prediction. Of course, the performance of neural networks can be determined by factors like the quality of the sample, the network structure, and the training parameters [9]. Therefore, in this study, hybrid methods of artificial neural networksimperialist competitive algorithm (ANN-ICA) and artificial neural networks- gray wolf optimization (ANN-GWO) are applied. Following these methods are explained.

\section{A. Artificial Neural Network (ANN)}

The ANN is a machine learning method that explores the relationship between phenomena (input-output data pairs) by benchmarking the human brain process in the problemsolving processes [9]. There are three layers in the ANN method input, hidden and output in which the connection among the layers provides the possibility of connecting every single neuron in one layer to all possible neurons in the other layer. The selections of and the design of meta-parameters such as learning rate, output function, number of nodes in the hidden layer, and inputs are very determinant in the accuracy and performance of the ANN [10].

In this method, the dataset is split into three categories, training, validation, and testing. The ANN learns interaction among input and out pairs by finding the patterns using algorithms such as back-propagation and optimization models
[9], in the training phase. It is necessary to validate the datasets for adjusting and increase the accuracy of the learning process. And the prediction power of the developed ANN model is evaluated using the testing dataset and after the training phase.

\section{B. Imperialist Competitive Algorithm}

The imperialist competitive algorithm is a well-known optimization algorithm [11] proposed by Atashpai Gargari and Lucas [12]. Atashpai Gargari and Lucas [12] inspired by imperialistic competition develop a method so-called imperialist competitive algorithm [13] in which the countries classified into two groups based on their power: 1) colonies and 2) imperialists. Where one empire includes an imperialist with its colonies. The empires tend to widen their territories by controlling more colonies from the other empires. This makes competition among the empires in which the strongest empire dominates and controls the weaker colonies [14].

\section{Hybrid ANN-ICA Method}

Through hybridization of ANNs the parameters of the model can be optimized using an efficient optimization method. Here, the ICA is used to tune ANN meta-parameters optimally to improve the model accuracy [15]. In other words, ICA helps the ANN to optimize the weights and biases. As a result, the error rate decreases, and the performance of the model in prediction increases. In the literature, eight steps are introduced to perform an ICA: 1) defining an initial empire, 2) determining the positions of the imperialist and colonies, 3 ) specifying the power of an empire, 4) identifying the competition among the empires, 5) omitting the weaker empires, 6) the convergence [16]. Thus, if the metaparameters (countries) are coded as the variables, then country $\mathrm{X}$ is defined as:

$$
X \in\left\{x_{1}, x_{2}, x_{3}, x_{4}\right\}
$$

The number of neurons in the hidden layer is shown as $x_{1}$. The $x_{1}$, can take a value of $1<x_{1}<100, x_{2}$ is the input layer, and $x_{3}$ is the output activation function. The value of $x_{1}$ and $x_{2}$ can vary from 1 to 5 and $x_{4}$ is the learning rate that can be between $0-5$.

\section{Gray Wolf Optimizer}

Mirjalili et al. [17] have developed the Gray Wolf Optimizer (GWO) algorithm inspired by the hunting process of gray wolves. Wolves usually lives in a group of 5 to 12 as two of them lead the group as it has formed a strong social hierarchy within the group. Mirjalili et al. [17] explain this hierarchy in a way that the alphas wolves $(\alpha)$ are the leader's group and make the decisions. The betas wolves $(\beta)$ are in the second level and they support the alphas wolves' decisions. The deltas wolves $(\delta)$ are in the next level and they are the followers of alpha and beta wolves. According to Mirjalili et al. [17], there are 5 types of deltas wolves: 1) Scouts who are responsible for controlling the boundaries of the territory, 2) Sentinels who are the group security and protect the group in case of danger, Elders who are young and very strong and they are the potential alpha or beta wolves, Hunters who assist the alpha and beta in hunting prey for the group, and Caretakers who look after the ill and wounded wolves. In the lowest level of this community are the omegas wolves $(\omega)$ who follow the superior wolves and they are the lasts allowing to eat. Accordingly, alpha $(\alpha)$ wolves are the most desirable solutions in the GWO algorithm and the other best solutions are considered Beta $(\beta)$ and delta $(\delta)$. In this methodology, the provided results in the group (populations) are omega $(\omega)$. 
Following the mathematical equations of the hunting, process is provided.

Alpha, beta and omega wolves respectively have the responsibility to guide hunting. They firstly circle the pray's first step of hunting prey is circling it by $\alpha, \beta$, and $\omega$. The mathematical model of the circling process as shown in equations $1,2,3$ and 4 .

$$
X(t+1)=X_{p}(t)+A \cdot D
$$

Where $\mathrm{X}$ is the representation of the gray wolf position, $\mathrm{t}$ is the number of iterations, $\mathrm{Xp}$ is the prey position and finally, A and D can be calculated by (3) and (4).

In equation 4 , represents the number of iterations, which varies from 0 to 2 . NumIter refers to the total number of iterations. In addition, $\mathrm{r} 1$ and $\mathrm{r} 2$ are random vectors between $[0,1]$ simulating the hunting. Equation 7 shows the update of wolves' positions.

$$
\begin{gathered}
D=\left|C \cdot X_{p}(t+1)-X(t)\right| \\
A=2 a \cdot r_{1}-a \\
C=2 r_{2} \\
a=2-t(2 / \text { NumIter }) \\
X(t+1)=\left(X_{1}+X_{2}+X_{3}\right) / 3
\end{gathered}
$$

$\mathrm{X}_{1}, \mathrm{X}_{2}$, and $\mathrm{X}_{3}$ can be calculated as follow:

$$
\begin{aligned}
X_{1} & =\left[X_{\alpha}-A_{1} \cdot D_{\alpha}\right] \\
X_{2} & =\left[X_{\beta}-A_{2} \cdot D_{\beta}\right] \\
X_{3} & =\left[X_{\delta}-A_{3} \cdot D_{\delta}\right] \\
D_{\alpha} & =\left[C_{1} \cdot X_{\alpha}-X\right] \\
D_{\beta} & =\left[C_{2} \cdot X_{\beta}-X\right] \\
D_{\delta} & =\left[C_{3} \cdot X_{\delta}-X\right]
\end{aligned}
$$

It can be interpreted that $\mathrm{X}_{1}, \mathrm{X}_{2}$, and $\mathrm{X}_{3}$ are considered as the best solutions at $t$ iteration. $A_{1}, A_{1}$, and $A 3$ can be calculated by equation 3 . And $\mathrm{C}_{1}, \mathrm{C}_{2}$, and $\mathrm{C}_{3}$ are measured by equation 4.

\section{E. Gray Wolf Optimizer of Neural Networks}

In the gray wolf optimizer of neural networks (ANNGWO) firstly, GWO trains the ANN to optimize the initial weight and biases. Then the neural network will be trained by the back-propagation algorithm to tune the weights and biases calculated in the previous stage in order to find the most global optima model.

\section{F. Accuracy metrics}

The next step modeling the machine learning methods is to test the accuracy. The model with the lowest error level and highest correlation will be selected as the best model. To assess the accuracy performance of the models of ANN-ICA and ANN-GWO, two metrics of Root mean square error (RMSE) and the relative mean absolute error [12] are selected. To measure the correlation, the metrics of correlation coefficient (R) is used. Equations 14, 15, and 16 explained these metrics in detail.

$$
R M S E=\sqrt{\frac{1}{N} \sum_{i=1}^{N}(A-P)^{2}}
$$

$$
\begin{gathered}
R=\left(1-\left(\frac{\sum_{i=1}^{n}(A-P)^{2}}{\sum_{i=1}^{n} A_{i}^{2}}\right)\right)^{1 / 2} \\
M A E=\frac{\sum_{i=1}^{n}|A-P|}{N}
\end{gathered}
$$

Where $\mathrm{A}$ is the target value, $\mathrm{P}$ is the predicted values, and $\mathrm{N}$ is the numbers of data.

\section{RESULTS AND DISCUSSION}

As it is mentioned above, RMSE, MEA, and R metrics applied to test the accuracy of the ANN-ICA and ANN-GWO in the crop-yield prediction. As it is presented at the bottom of table II, the average RMSE of ANN-ICA (3.20) which is slightly higher than ANN-GWO (3.19). These figures indicate that, in terms of these two metrics, the performance of ANNGWO in the crop yield production has been better than ANNICA. The best result per crop is highlighted in bold in table II.

Besides, the results for the metric of R depicted in Fig. 2. Here best result per crop is highlighted in bold in the table as well. Since the average metric of $\mathrm{R}$ is higher for ANN-GWO (0.48) method than ANN-ICA (0.42) it is interpreted that the performance of ANN-GWO in this metric has been better. In addition, the average MEA for ANN-ICA (27.22) is higher than ANN-GWO (26.65) which represents the better performance of ANN-GWO in these metrics. According to table II and the R metric, an individual counting of best results reveals that ANN-GWA gets most of the best-correlated models (three models).

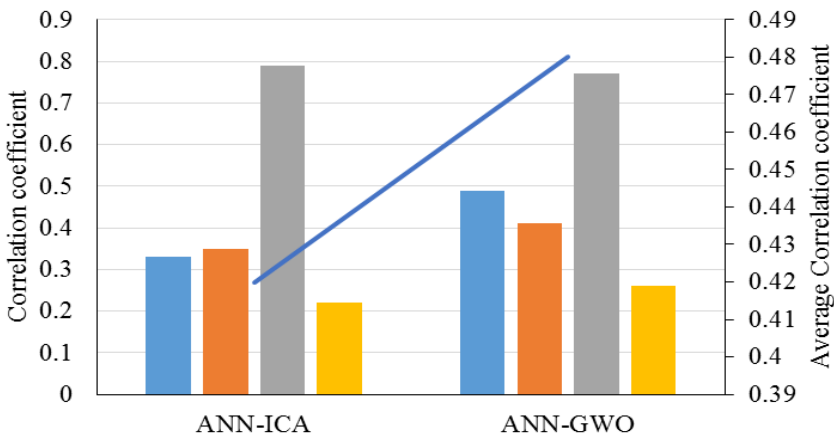

Wheat Barley Potato Sugar Beet $\longrightarrow$ Average

Fig. 2. Comparison of correlation coefficient of ANN-ICA and ANNGWO per each crop.

Since selected attributes affect the model performance [18] the importance of each attribute in the performance of the selected methods (ANN-ICA and ANN-GWO) is measured and presented in table III.

To simplify presenting the attributes in the table, each attribute gets a code. Attribute code for attributes planting area is AT1, for irrigation water depth is AT2, for rainfall during the crop growth stages is AT3, for global solar radiation is AT4, for maximum temperatures is AT5, for average temperatures is AT6, and for minimum temperatures is AT7. One of our contributions to the current research is that the attributes are compared, and the best attribute set is identified for each technique. Table III exposes the lack of consistency among the best attribute sets chosen by the methods as there is no evidence of proving the preference of an individual model to constitute a set of attributes. Whilst, there is in the 
set of attributes by the methods related to the crops. For example, for all the crops AT2 are included regardless of the methods.

TABLE II. EVALUATION OF THE ACCURACY OF ANN-ICA AND ANN-GWO WITH R, MEA, AND RMSE

\begin{tabular}{|c|c|c|c|c|c|c|}
\hline \multirow{2}{*}{ Crops } & \multicolumn{2}{|c|}{ R } & \multicolumn{2}{c|}{ MEA (\%) } & \multicolumn{2}{c|}{ RMSE (\%) } \\
\cline { 2 - 7 } & $\begin{array}{c}\text { ANN- } \\
\text { ICA }\end{array}$ & $\begin{array}{c}\text { ANN- } \\
\text { GWO }\end{array}$ & $\begin{array}{c}\text { ANN- } \\
\text { ICA }\end{array}$ & $\begin{array}{c}\text { ANN- } \\
\text { GWO }\end{array}$ & $\begin{array}{c}\text { ANN- } \\
\text { ICA }\end{array}$ & $\begin{array}{c}\text { ANN- } \\
\text { GWO }\end{array}$ \\
\hline Wheat & 0.33 & $\mathbf{0 . 4 9}$ & 35.04 & $\mathbf{3 3 . 3 0}$ & 8.48 & $\mathbf{8 . 4 1}$ \\
\hline Barley & 0.35 & $\mathbf{0 . 4 1}$ & $\mathbf{1 2 . 0 7}$ & 12.13 & $\mathbf{0 . 3 2}$ & 0.33 \\
\hline Potato & $\mathbf{0 . 7 9}$ & 0.77 & 22.76 & $\mathbf{2 2 . 1 5}$ & 0.68 & $\mathbf{0 . 6 6}$ \\
\hline $\begin{array}{c}\text { Sugar } \\
\text { Beet }\end{array}$ & 0.22 & $\mathbf{0 . 2 6}$ & $\mathbf{3 8 . 9 9}$ & 39.02 & $\mathbf{3 . 3 2}$ & 3.34 \\
\hline Average & 0.42 & 0.48 & 27.22 & 26.65 & 3.20 & 3.19 \\
\hline
\end{tabular}

TABLE III. THE EFFECT OF ATTRIBUTES ON THE ANN-ICA AND ANN-WGO MODELS

\begin{tabular}{|c|c|c|c|c|c|c|c|c|}
\hline \multirow[b]{2}{*}{ Crops } & \multirow[b]{2}{*}{ Method } & \multicolumn{7}{|c|}{ Attributes } \\
\hline & & $\begin{array}{c}\text { AT } \\
1\end{array}$ & $\begin{array}{c}\text { AT } \\
2\end{array}$ & $\begin{array}{c}\text { AT } \\
\mathbf{3}\end{array}$ & $\begin{array}{c}\text { AT } \\
4 \\
\end{array}$ & $\begin{array}{c}\text { AT } \\
5\end{array}$ & $\begin{array}{c}\text { AT } \\
6\end{array}$ & $\begin{array}{c}\text { AT } \\
7 \\
\end{array}$ \\
\hline \multirow[b]{2}{*}{ Wheat } & ANN-ICA & 0 & 1 & 3 & 1 & 0 & 0 & 1 \\
\hline & $\begin{array}{l}\text { ANN- } \\
\text { GWO }\end{array}$ & 0 & 3 & 0 & 2 & 2 & 0 & 1 \\
\hline \multirow[b]{2}{*}{ Barley } & ANN-ICA & 1 & 3 & 1 & 2 & 1 & 1 & 0 \\
\hline & $\begin{array}{l}\text { ANN- } \\
\text { GWO }\end{array}$ & 0 & 2 & 1 & 1 & 3 & 1 & 0 \\
\hline \multirow[b]{2}{*}{ Potato } & ANN-ICA & 3 & 3 & 3 & 0 & 3 & 3 & 2 \\
\hline & $\begin{array}{l}\text { ANN- } \\
\text { GWO }\end{array}$ & 3 & 3 & 3 & 0 & 1 & 1 & 2 \\
\hline \multirow[b]{2}{*}{$\begin{array}{c}\text { Sugar } \\
\text { Beet }\end{array}$} & ANN-ICA & 2 & 3 & 2 & 1 & 0 & 1 & 2 \\
\hline & $\begin{array}{l}\text { ANN- } \\
\text { GWO }\end{array}$ & 2 & 3 & 0 & 2 & 1 & 3 & 0 \\
\hline
\end{tabular}

\section{CONCLUSION}

Various approaches are taken by researchers to predict the crop yield, such as regression models and machine learning methods. The main contribution of the current study and comparison of two hybrid machine learning methods for the prediction of crop yield, for the first time in the literature. One of the most important reasons that hybrid machine learning methods are considered is that the accuracy of the prediction of such models is higher. On the other hand, in this study, numerous attributes are considered to evaluate the performance of the models. For this purpose, firstly, the best attributes set for each method were identified among the potential attributes. According to the result the ANN-GWO method with R of 0.48 , RMSE of 3.19, and MEA of 26.65 had a better performance in the crop yield prediction. Since, a different set of attributes affect the performance of the model, it is recommended that future research examine a different set of attributes and compare the results. Besides, it is also recommended to compare other hybrid machine learnings to find the proper model. For the future research, advancement of hybrid and ensemble machine learning models, e.g., [1924], and comparative analysis with deep learning models, e.g., [25-28] are proposed to identify models with higher efficiency.

\section{ACKNOWLEDGMENT}

We acknowledge the financial support of this work by the Hungarian State and the European Union under the EFOP3.6.1-16-2016-00010 project and the 2017-1.3.1-VKE-201700025 project.

\section{REFERENCES}

[1] Ericksen, P.J., J.S. Ingram, and D.M. Liverman, Food security and global environmental change: emerging challenges. 2009, Elsevier.

[2] Nair, S.S., et al., Importance of Crop Yield in Calibrating Watershed Water Quality Simulation Tools 1. JAWRA Journal of the American Water Resources Association, 2011. 47(6): p. 12851297.

[3] Prasad, A.K., et al., Crop yield estimation model for Iowa using remote sensing and surface parameters. International Journal of Applied Earth Observation and Geoinformation, 2006. 8(1): p. 2633.

[4] Khairunniza-Bejo, S., S. Mustaffha, and W.I.W. Ismail, Application of artificial neural network in predicting crop yield: A review. Journal of Food Science and Engineering, 2014. 4(1): p.

[5] Schlenker, W. and M.J. Roberts, Estimating the impact of climate change on crop yields: The importance of nonlinear temperature effects. 2008, National Bureau of Economic Research.

[6] Irmak, A., et al., Artificial neural network model as a data analysis tool in precision farming. Transactions of the ASABE, 2006. 49(6): p. 2027-2037.

[7] Tsai, C.-F. and M.-L. Chen, Credit rating by hybrid machine learning techniques. Applied soft computing, 2010. 10(2): p. 374380

[8] Drummond, S.T., et al., Statistical and neural methods for sitespecific yield prediction. Transactions of the ASAE, 2003. 46(1): p. 5 .

[9] Rem, B.S., et al., Identifying quantum phase transitions using artificial neural networks on experimental data. Nature Physics, 2019. 15(9): p. 917-920.

[10] Sajan, K., V. Kumar, and B. Tyagi. ICA based Artificial Neural Network model for voltage stability monitoring. in TENCON 2015-2015 IEEE Region 10 Conference. 2015. IEEE.

[11] Al Dossary, M.A. and H. Nasrabadi, Well placement optimization using imperialist competitive algorithm. Journal of Petroleum Science and Engineering, 2016. 147: p. 237-248.

[12] Atashpaz-Gargari, E. and C. Lucas. Imperialist competitive algorithm: an algorithm for optimization inspired by imperialistic competition. in 2007 IEEE congress on evolutionary computation. 2007. IEEE.

[13] Bruzzone, A.G., et al., Simulation Based Design of Innovative Quick Response Processes in Cloud Supply Chain Management for "Slow Food" Distribution, in Theory, Methodology, Tools and Applications for Modeling and Simulation of Complex Systems. 2016, Springer. p. 25-34.

[14] Zhang, Z., et al., Development of an adaptive relevance vector machine approach for slope stability inference. Neural Computing and Applications, 2014. 25(7-8): p. 2025-2035.

[15] Gao, W., et al., A predictive model based on an optimized ANN combined with ICA for predicting the stability of slopes. Engineering with Computers, 2019: p. 1-20.

[16] Le, L.T., et al., A comparative study of PSO-ANN, GA-ANN, ICA-ANN, and ABC-ANN in estimating the heating load of buildings' energy efficiency for smart city planning. Applied Sciences, 2019. 9(13): p. 2630.

[17] Mirjalili, S., S.M. Mirjalili, and A. Lewis, Grey wolf optimizer. Advances in engineering software, 2014. 69: p. 46-61.

[18] Rezakazemi, M., Mosavi, A. and Shirazian, S., ANFIS pattern for molecular membranes separation optimization. Journal of Molecular Liquids, 2019, 274, pp.470-476.

[19] Ardabili, S., Mosavi, A., and Várkonyi-Kóczy, A.R.: Advances in Machine Learning Modeling Reviewing Hybrid and Ensemble Methods, 2020, Lecture Notes in Networks and Systems 101, pp. 215-227.

[20] Ardabili, S., Mosavi, A., and Várkonyi-Kóczy, A.R., Systematic Review of Deep Learning and Machine Learning Models in Biofuels Research, 2020, Lecture Notes in Networks and Systems 101, pp. 19-32.

[21] Gundoshmian, T.M., Ardabili, S., Mosavi, A., and VárkonyiKóczy, A.R. Prediction of Combine Harvester Performance Using Hybrid Machine Learning Modeling and Response Surface 
Methodology, 2020, Lecture Notes in Networks and Systems 101, pp. 345-360

[22] Joloudari, J.H., Joloudari, E.H., Saadatfar, H., Ghasemigol, M., Razavi, S.M., Mosavi, A., Nabipour, N., Shamshirband, S., and Nadai, L. Coronary artery disease diagnosis; ranking the significant features using a random trees model, Int. J. Environ. Res. Public Health, 2020, 17, (3)5.

[23] Nosratabadi, S., Mosavi, A., Keivani, R., Ardabili, S., and Aram, F. State of the Art Survey of Deep Learning and Machine Learning Models for Smart Cities and Urban Sustainability, 2020, Lecture Notes in Networks and Systems 101, pp. 228-238

[24] Ardabili, S., Mosavi, A., Dehghani, M., and Várkonyi-Kóczy, A.R. Deep Learning and Machine Learning in Hydrological Processes Climate Change and Earth Systems a Systematic Review, 2020, Lecture Notes in Networks and Systems 101, pp. $52-62$.

[25] Ardabili, S., Mosavi, A., Mahmoudi, A., Gundoshmian, T.M., Nosratabadi, S., and Várkonyi-Kóczy, A.R. Modelling Temperature Variation of Mushroom Growing Hall Using Artificial Neural Networks, 2020, Lecture Notes in Networks and Systems 101, pp. 33-45.

[26] Mosavi, A., Ozturk, P., \& Chau, K. W. Flood prediction using machine learning models: Literature review. Water, 2018, 10 (11), 1536.

[27] Ardabili, S., Mosavi, A., and Várkonyi-Kóczy, A.R. Building Energy Information: Demand and Consumption Prediction with Machine Learning Models for Sustainable and Smart, 2020, Lecture Notes in Networks and Systems101, pp. 191-201.

[28] Mosavi, A., Ardabili, S., and Várkonyi-Kóczy, A.R. List of Deep Learning Models, 2020, Lecture Notes in Networks and Systems 101, pp. 202-214. 